\title{
ADVANCED SOLIDS NMR STUDIES OF COAL STRUCTURE AND CHEMISTRY
}

\section{PROGRESS REPORT}

including

the period September 1, 1996 to March 1, 1997

Kurt W. Zilm, PI

Department of Chemistry

Yale University

New Haven, CT 06511

Prepared for the Department of Energy

Agreement No. DE-FG22-95PC95227 -04

Granting period: 9/1/95-8/31/98

Award: \$200,000/3 yrs., \$63,116 current year

Program Manager: Richard F. Sprecher

COR: Suzanne K. Polmar 


\section{NOTICE}

This report was prepared as an account of work sponsored by the United States Government. Neither the United States nor the Department of Energy, nor any of their employees, nor any of their contractors, subcontractors, or their employees, make any warranty, express or implied, or assumes any legal liability or responsibility for the accuracy, completeness, or usefulness of any information, apparatus, product or process disclosed or represents that its use would not infringe on privately-owned rights.

US/DOE Patent Clearance is not required prior to publication of this document. 
This report covers the progress made on the title project for the project period. The study of coal chemical structure is a vital component of research efforts to develop better chemical utilization of coals, and for furthering our basic understanding of coal geochemistry. In this grant we are addressing several structural questions pertaining to coals with advances in state of the art solids NMR methods. Our goals are twofold. First, we are interested in developing new methods that will enable us to measure important structural parameters in whole coals not directly accessible by other techniques. In parallel with these efforts we will apply these NMR methods in a study of the chemical differences between gas-sourcing and oil-sourcing coals. The NMR methods work will specifically focus on determination of the number and types of methylene groups, determination of the number and types of methine groups, identification of carbons adjacent to nitrogen and sites with exchangeable protons, and methods to more finely characterize the distribution of hydrogen in coals. The motivation for investigating these specific structural features of coals arises from their relevance to the chemical reactivity of coals, and their suitability for possible correlations with the oil sourcing potential of some types of coals. The coals to be studied and contrasted include oil-prone coals from Australia and Indonesia, those comprising the Argonne Premium Coal Sample bank, and other relevant samples.

In this report period we have focused our work on 1 segment of the program. Our last report outlined progress in using our NMR editing methods with higher field operation where higher magic angle spinning rates are required. Significant difficulties were identified, and these have been the main subject of study during the most recent granting period.

\section{Progress on NMR methods Development}

The majority of our spectral editing work on coals has made use of a low field solids spectrometer which operates at $100 \mathrm{MHz}$ for protons. To be more widely applicable, we began work on making these methods work reliably at higher fields, i.e. $300 \mathrm{MHz}$ and above. This is motivated by the superior sensitivity available at higher fields, and the more widespread availability of spectrometers that operate at $300 \mathrm{MHz}$ than at $100 \mathrm{MHz}$. The principal difficulties involve accommodating higher sample spinning rates and the problems that accompany them in cross polarization $(\mathrm{CP})$ dynamics. At rates over $10 \mathrm{kHz}, \mathrm{CP}$ dynamics become more complex, and our editing protocols need modification. As a first step the Hartmann-Hahn match must be made under a sideband condition where $\omega_{1}=\omega_{2}+\omega_{1}$.

In the following we outline progress made on this problem. A new editing protocol has been developed for use at much higher spin rates. The work reported below was the subject of a poster presentation by one of the Ph.D. students on the project, Sean Burns, at the most recent Experimental NMR Conference in Orlando. Sean received a graduate student travel award from the conference for his excellent presentation.

\section{$\underline{\text { Background }}$}

Several spectral editing methods have been developed for assigning complex ${ }^{13} \mathrm{C}$ CPMAS solids NMR spectra. These methods separate spectra into subspectra based on the number of protons bonded to the various carbon centers. Protocols designed in our laboratory have used the differences in cross polarization (CP) dynamics among $\mathrm{C}, \mathrm{CH}, \mathrm{CH}_{2}$ and $\mathrm{CH}_{3}$ groups. An example of this approach is depicted in Figure 1, where the ${ }^{13} \mathrm{C}$ CPMAS spectrum of cholesteryl acetate has been separated into $\mathrm{C}, \mathrm{CH}, \mathrm{CH}_{2}$ and $\mathrm{CH}_{3}$ subspectra. 


\title{
Figure 1: Cholesteryl acetate 4kHz MAS.
}

\author{
difference
}
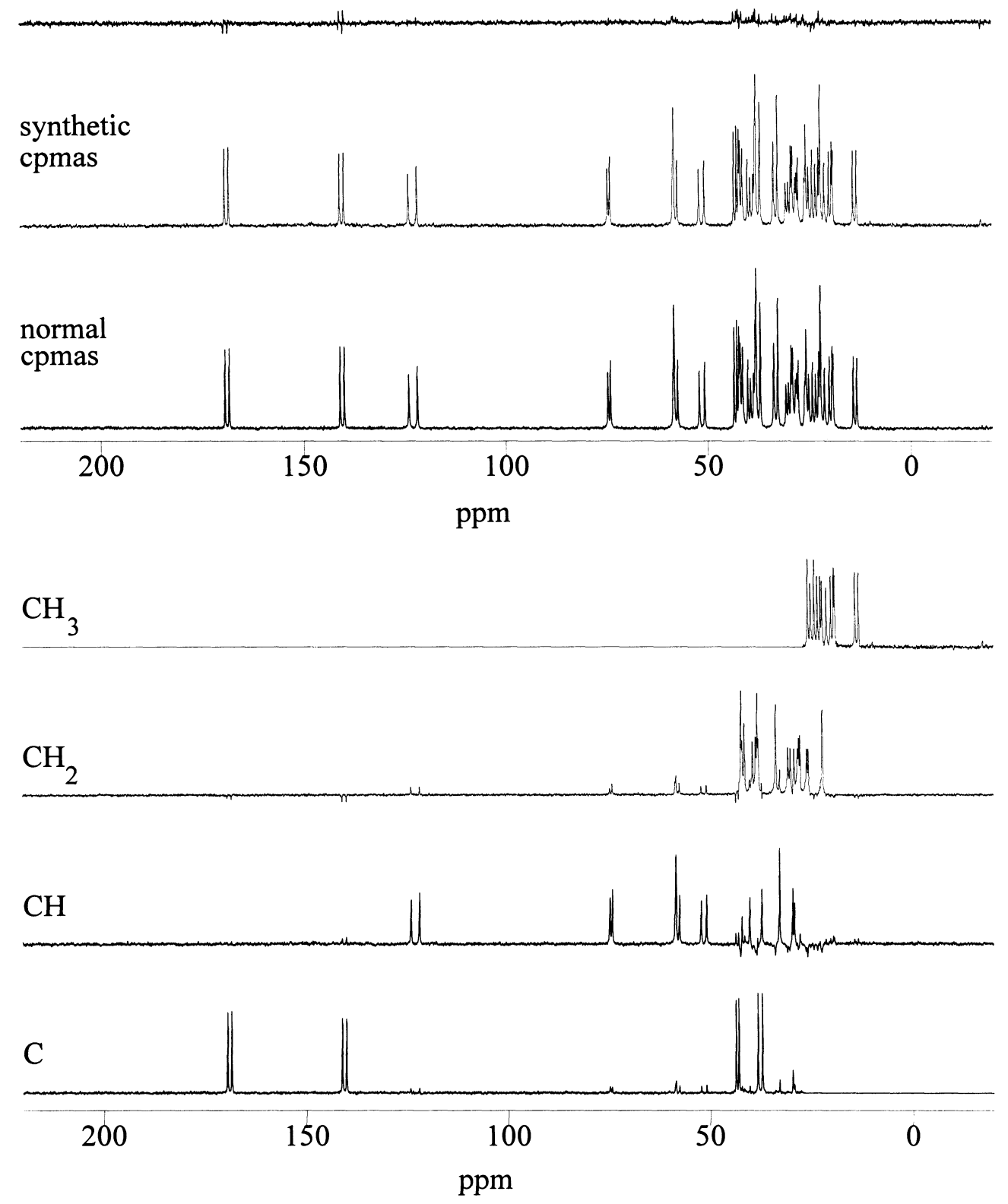

At moderate field strengths where MAS rates of $4 \mathrm{kHz}$ or less are sufficient, this is straightforward to accomplish using the $\mathrm{CP}$ dynamics characteristic of the standard Hartmann-Hahn match. Under these conditions $\mathrm{CH}$ and $\mathrm{CH}_{2}$ groups polarize quickly to a quasi-equilibrium state. In this state the initial spinlocked proton magnetization is distributed equally between the ${ }^{13} \mathrm{C}$ nucleus and its directly attached protons. Therefore a $\mathrm{CH}$ group is polarized to half intensity while a $\mathrm{CH}_{2}$ achieves two thirds of full intensity. The collection of pulse sequences making up our editing method basically play variations on this theme. Each sequence produces a spectrum in which the intensities of the resonances for carbons of differing multiplicities are modulated in distinct fashions. Unique linear combinations of these spectra produce the various subspectra. 
The use of a short CP mixing time in a standard CP sequence (Figure 2a) produces a spectrum dominated by $\mathrm{CH}$ and $\mathrm{CH}_{2}$ groups with predictable relative intensities which we call an SCP spectrum. Appending a short polarization inversion (PI) interval to the SCP sequence (Figure 2b) produces an SCPPI spectrum consisting solely of resonances from $\mathrm{CH}_{2}$ groups. Nonprotonated and $\mathrm{CH}_{3}$ carbons are preferentially detected in the LCPD experiment (Figure 2c) which consists of a long mixing time CP experiment (LCP) followed by a depolarization (D) interval.

c)

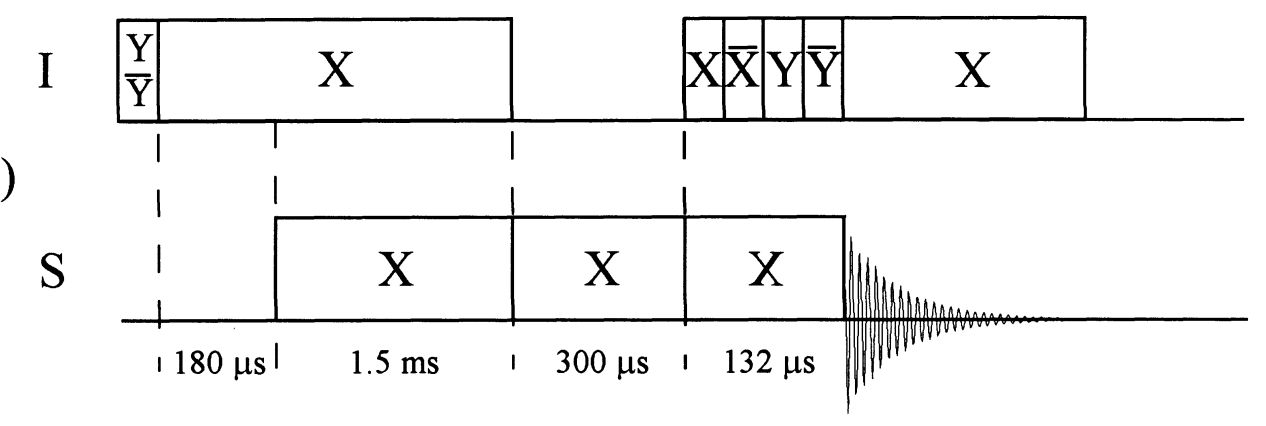

b)

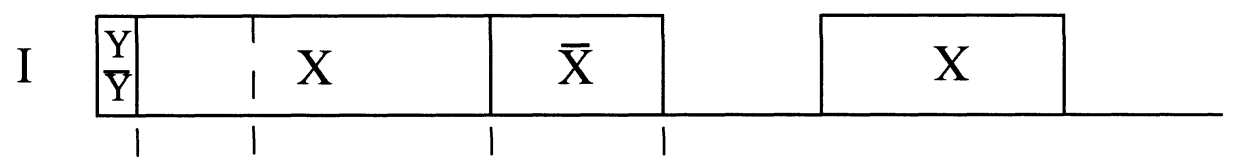

a)
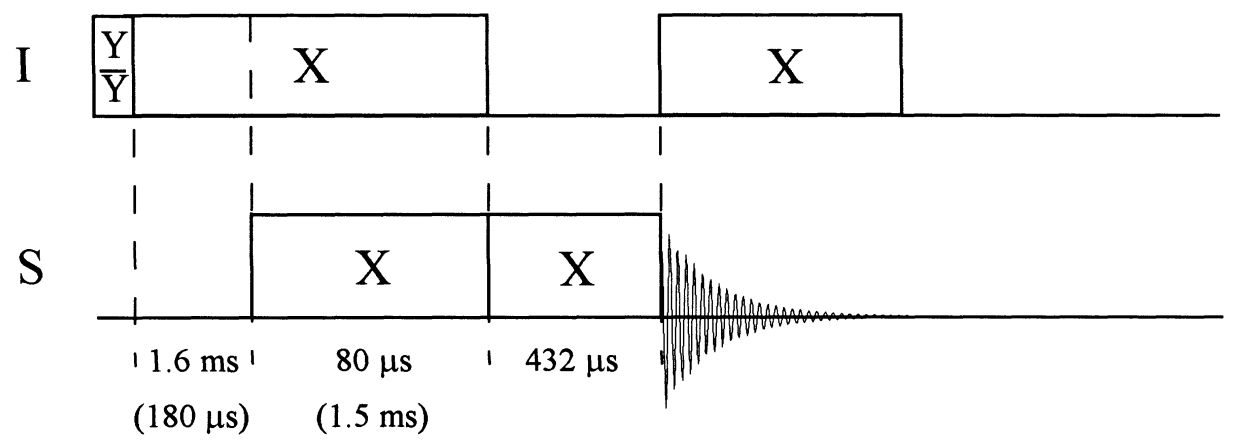

Figure 2: a) SCP (or LCP) sequence; b) SCPPI sequence; and c) LCPD sequence. 
Editing techniques based on these pulse sequences have two fundamental limitations. One is the need for a separation of the time scales between the initial transient polarization transfer and the long time approach of the ${ }^{13} \mathrm{C} \mathrm{CP}$ signal to its fully polarized state. The latter proceeds at a rate determined by spin diffusion among the abundant protons. Suppression of spin diffusion during CP should then potentially enable production of the quasi-equilibrium polarization condition with less sensitivity to the exact timing of the short CP transfers used. A simple method to accomplish CP transfer with suppression of ${ }^{1} \mathrm{H}-{ }^{1} \mathrm{H}$ spin diffusion is to perform the Hartmann-Hahn transfer with the protons spinlocked along an effective field tilted at the magic angle in the rotating frame. One approach to this is provided by the pulse sequence shown in Figure 3.

Another limitation of the present method is the need for efficient polarization transfer at the normal Hartmann-Hahn or centerband match condition, $\omega_{1}=\omega_{2}$. At the spin rates required to work at fields of $7 \mathrm{~T}$ or more, $\mathrm{CP}$ of nonprotonated carbon sites on a centerband match is futile. Therefore, at higher fields, a sideband match $\omega_{1}=\omega_{2} \pm \omega_{\mathrm{r}}$ is required. Both of these questions are investigated in the experiments described herein.

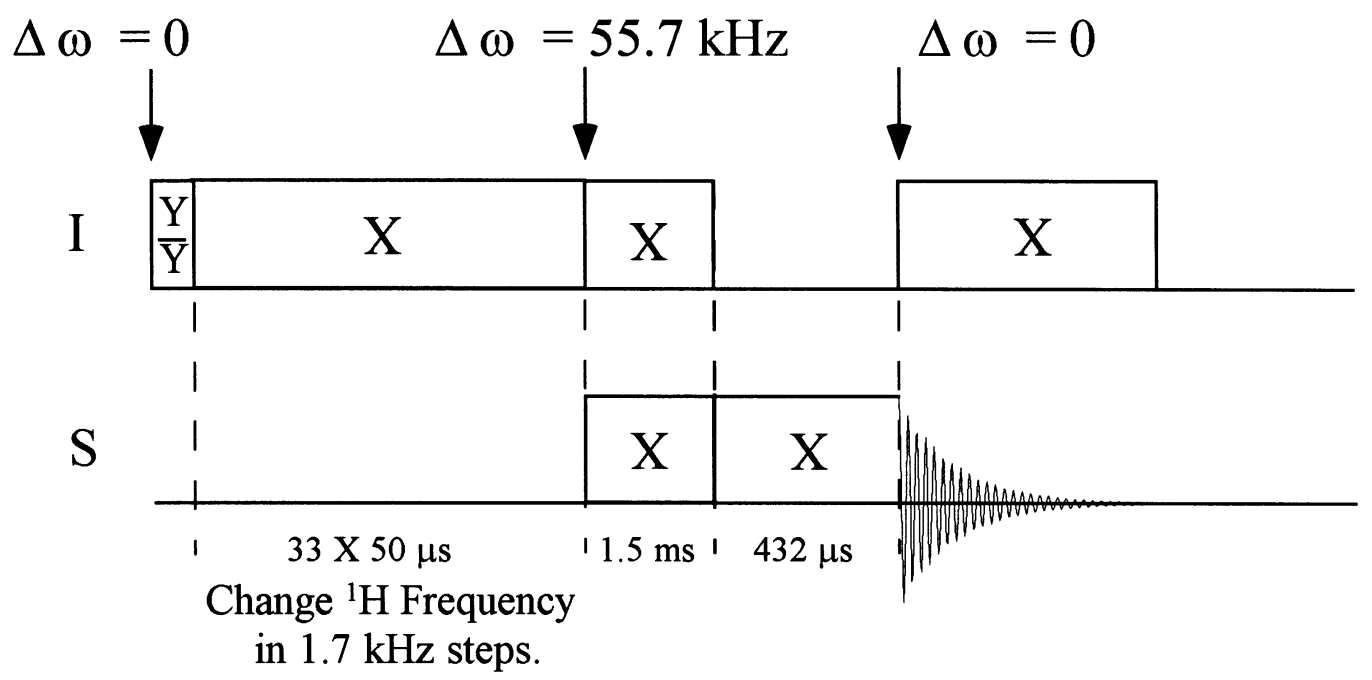

Figure 3: Sequence to spin lock the protons at the magic angle in order to suppress spin diffusion during $\mathrm{CP}$. 


\section{Experimental}

All experiments depicted were performed on a home built NMR spectrometer based on a Tecmag Libra data system and using an Oxford Instruments $7.05 \mathrm{~T}, 89 \mathrm{~mm}$ room-temperature bore superconducting solenoid. A 5mm double resonance CPMAS probe from Doty Scientific was used. Samples were spun at the magic angle at $12.0 \mathrm{kHz}$. Proton decoupling was accomplished at $3.0 \mathrm{ppm}$ with an $86 \mathrm{kHz}$ decoupling field. All samples were restricted to the middle of the rotor by packing the ends of the rotor with silica. This resulted in a cylindrical sample shape with a length of $6 \mathrm{~mm}$ and a diameter of 3.6 $\mathrm{mm}$. The Hartmann-Hahn sideband match condition used a ${ }^{13} \mathrm{C} \mathrm{RF}$ field amplitude $v^{\mathrm{C}}{ }_{1}=v^{\mathrm{H}}{ }_{1}+v_{\mathrm{r}}=73 \mathrm{kHz}$. The match was determined by maximizing the ${ }^{13} \mathrm{C}$ signal for adamantane while adjusting the ${ }^{13} \mathrm{C}$ RF field strength.

Magic angle spin locking experiments were performed by first spin locking the protons on resonance and then sweeping the frequency off resonance with a Sciteq direct digital frequency synthesizer (DDS). After an on resonance spinlock, the proton RF field was swept off resonance in 33 steps of 1.6893 $\mathrm{kHz}$ each at $50 \mu$ s intervals. In order to verify that the effective proton field was indeed at the magic angle, an off-resonance-decoupled spectrum of adamantane was acquired. This spectrum was monitored as a function of the decoupler offset to find the magic angle condition by maximizing the resolution of the ${ }^{1} \mathrm{~J}_{\mathrm{CH}}$ coupled multiplets observed. For the magic angle spin locking experiments, the proton field strength was $83 \mathrm{kHz}$ and the offset frequency was $55.7 \mathrm{kHz}$. This effective field of $100 \mathrm{kHz}$ was matched to a carbon field of $88 \mathrm{kHz}$ at $12.0 \mathrm{kHz}$ spinning for the sideband match experiments. In centerband match experiments both RF fields were set at $100 \mathrm{kHz}$.

CP Results

$\mathrm{CP}$ buildup curves acquired using sequence $2 \mathrm{a}$ by varying the contact time are shown in Figures 4 and 5 for various conditions. In both cases the sample used was sodium hydroxybutyrate. Figure 4 illustrates the influence on the ${ }^{13} \mathrm{CH}$ signal intensity of high speed MAS and changing the angle of the effective proton spin locking field with respect to the static field. Data were acquired at $12 \mathrm{kHz}$ spinning with the effective field inclined at the magic angle (green and blue) and perpendicular (red) to the static field. Data acquired at $4 \mathrm{kHz}$ on a sideband match (purple) are included for comparison. From these curves, one can see that the $\mathrm{CP}$ buildup on a sideband match at $12 \mathrm{kHz}$ is slightly slower than at $4 \mathrm{kHz}$. The initial slope is further weakened by magic angle spin locking. MAS at $12 \mathrm{kHz}$ alone provides sufficient suppression of the ${ }^{1} \mathrm{H}-{ }^{1} \mathrm{H}$ spin diffusion that the initial transient polarization transfer sustains a long lived oscillation. The use of magic angle spinlocking in the $\mathrm{CP}$ transfer further enhances the oscillations and leads to a smaller equilibrium polarization.

These data indicate that the editing protocol developed previously for a centerband match at low MAS rates is ill-suited for higher spinning speeds. The quasi-equilibrium state is not easily identified under these conditions where the transient oscillation is better developed and longer lasting. Suppression of the ${ }^{1} \mathrm{H}-{ }^{1} \mathrm{H}$ spin diffusion only makes the situation worse. 


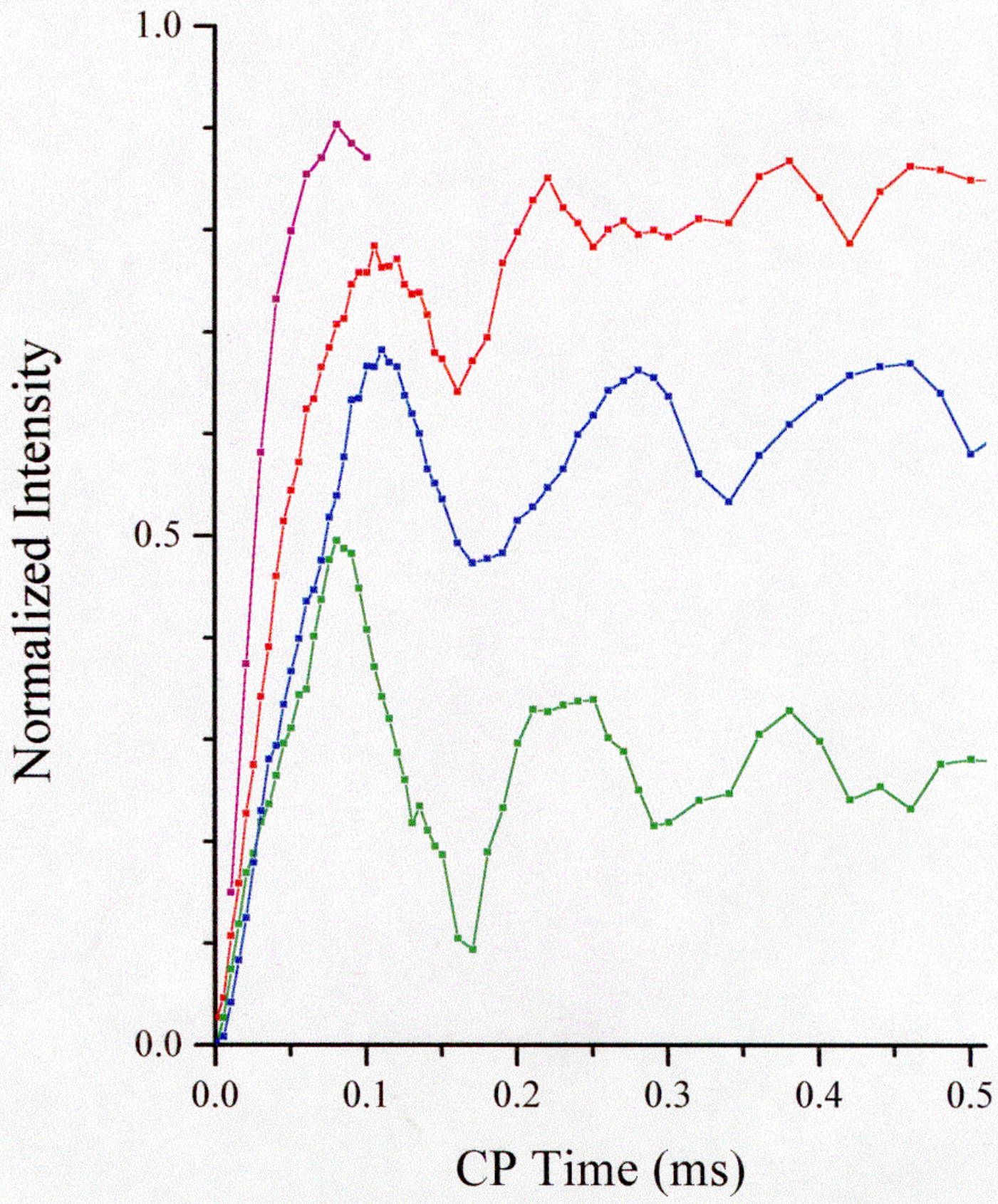

Figure 4: Methine signal as a function of $\mathrm{CP}$ time.

- $12 \mathrm{kHz}$ Normal Sideband Match

- $12 \mathrm{kHz}$ Magic Angle Centerband Match

- $12 \mathrm{kHz}$ Magic Angle Sideband Match

- $4 \mathrm{kHz}$ Normal Sideband Match 


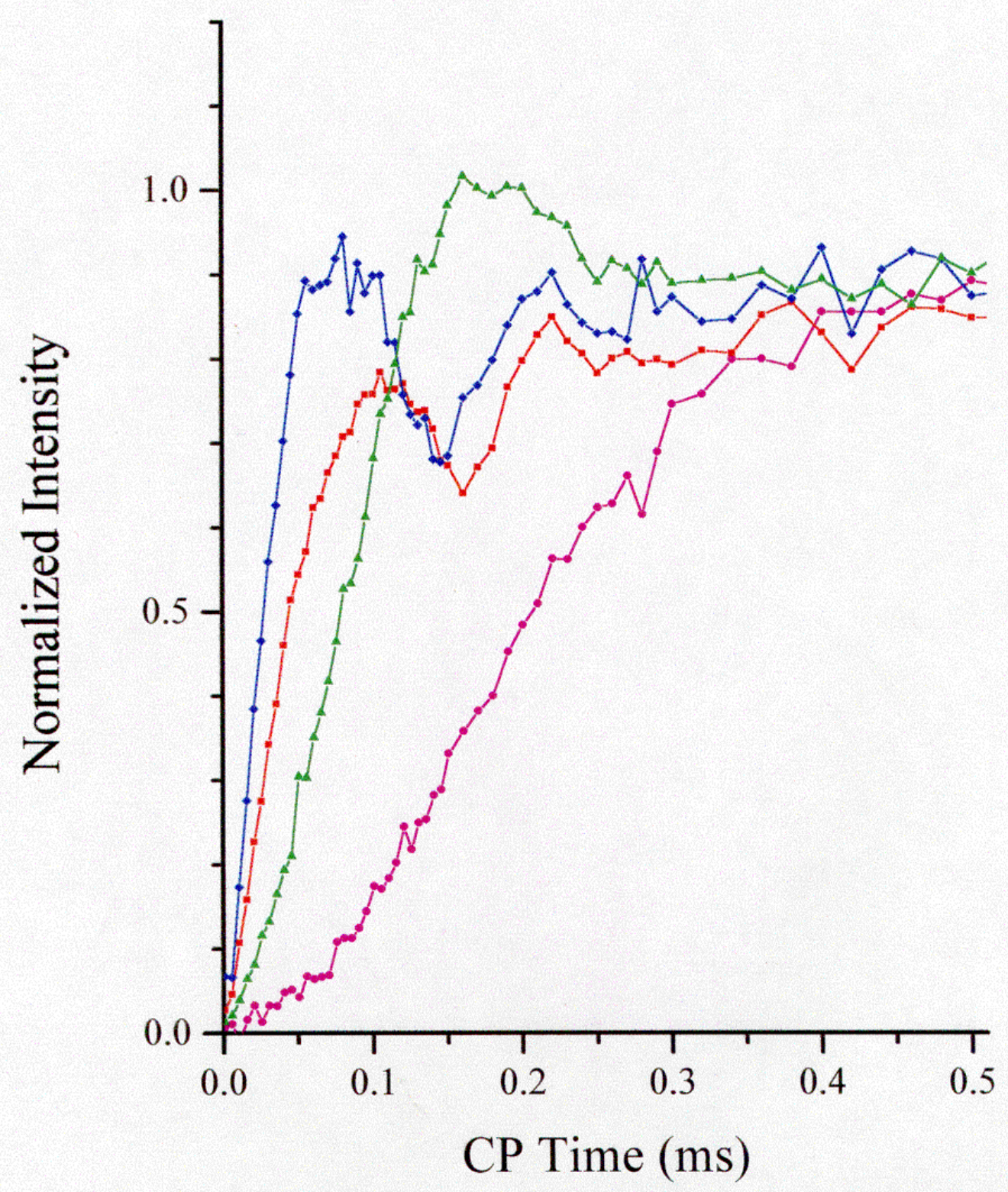

Figure 5: Time dependence of ${ }^{13} \mathrm{C}$ signals under sideband matched cross polarization for $12.0 \mathrm{kHz}$ MAS.

$\rightarrow-\mathrm{C}-; \rightarrow \mathrm{CH} ; \rightarrow \mathrm{CH}_{2} ; \rightarrow \mathrm{CH}_{3}$ 


\section{Development of a High Speed MAS Editing Protocol}

At first glance, the oscillatory time dependence of the $\mathrm{CH}, \mathrm{CH}_{2}$, and $\mathrm{CH}_{3}$ sideband match $\mathrm{CP}$ signals at higher spin rates would seem to interfere with the development of a spectral editing scheme. However, the oscillations occur primarily in the intermediate time regime. Our editing protocols rely on very short time $(<100 \mu \mathrm{s})$ and long time $(>1 \mathrm{~ms})$ behavior to highlight the differences in CP behavior of strongly and weakly coupled groups.

Of these three cross polarization methods, the normal sideband match experiment is most useful for editing. Figure 5 shows the intensities of each of the carbon types for the normal sideband match $\mathrm{CP}$ at $12.0 \mathrm{kHz}$. The $\mathrm{CH}$ and $\mathrm{CH}_{2}$ signal intensities are not sensitive to the $\mathrm{CP}$ time near $100 \mu$ s since the slope is zero. Around this point in the development of the $\mathrm{CP}$ buildup curves the $\mathrm{CH}$ and $\mathrm{CH}_{2}$ carbons have quite different polarizations. Using the $\mathrm{CP}$ behavior observed, a set of timings was chosen to provide good differential contrast between the various types of carbons while maintaining good sensitivity (see Figure 2). Long CP periods are set at $1.5 \mathrm{~ms}$, and short CP contacts at 60-80 $\mu$ s. Application of the set of pulse sequences and timings shown in Figure 2 on a $12 \mathrm{kHz}$ sideband match results in the spectra shown in Figure 6.

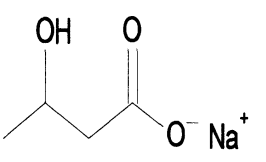

SCPPI
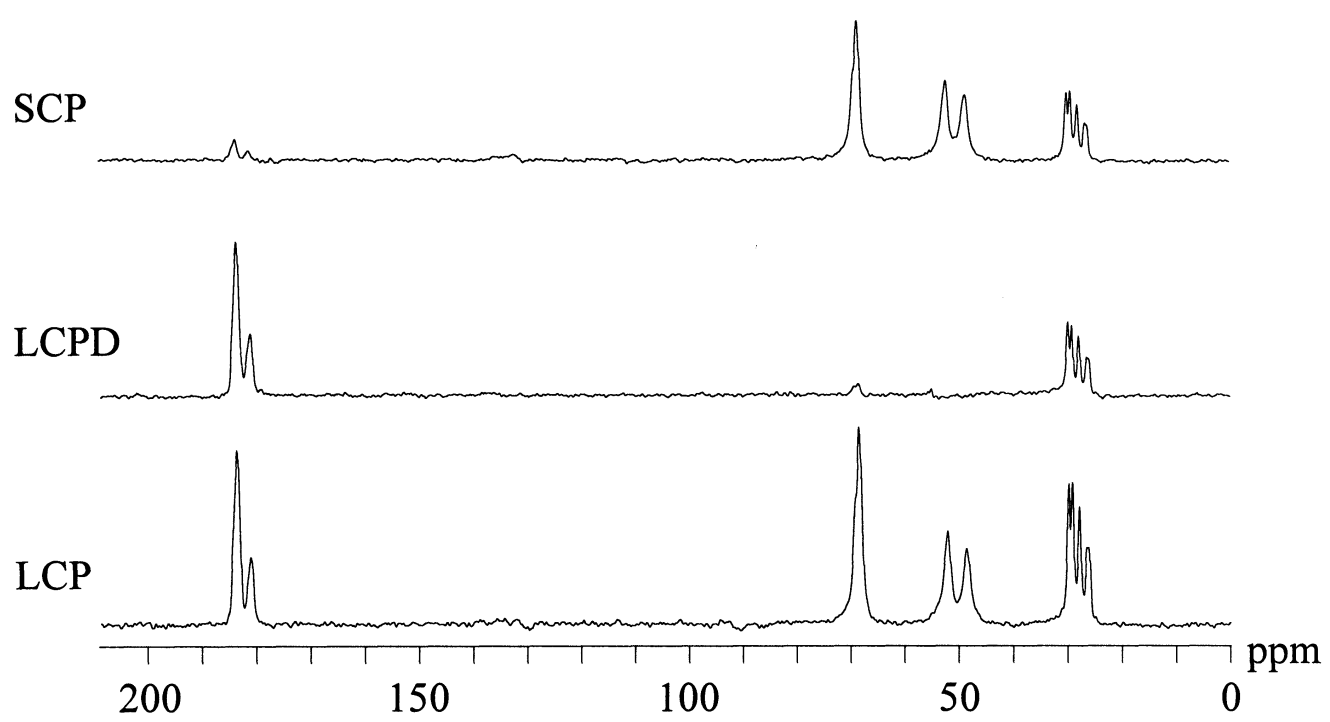

Figure 6: Sodium Hydroxybutyrate. 
The intensity matrix shown in Figure 7 was derived from this data and forms the basis of a high speed MAS editing protocol. It is similar to the matrix used in our original method. The LCP spectrum is taken as the intensity standard and the other spectra are measured relative to it. The SCP spectral intensities show a good difference between the tightly and weakly coupled groups. The $\mathrm{CH}$ and $\mathrm{CH}_{2}$ groups are more fully polarized than the nonprotonated or methyl carbons and exhibit larger intensity matrix elements. At $12 \mathrm{kHz}$ the signals are stronger than in the original method as a longer CP time of 80 $\mu \mathrm{s}$ is used instead of the previously used $40 \mu \mathrm{s}$. As in the original method, the SCPPI pulse sequence yields a $\mathrm{CH}_{2}$ only spectrum; in this case the $\mathrm{CH}_{2}$ intensity is -0.25 . Depolarization is not quite as efficient at this spinning speed and a longer depolarization time is needed to null the $\mathrm{CH}$ and $\mathrm{CH}_{2}$ signals (132 $\mu$ s). As a result of this, the $\mathrm{CH}_{3}$ signal in the LCPD spectrum is weaker.

\begin{tabular}{l|cccc} 
SPECTRUM & $-\mathrm{C}-$ & $\mathrm{CH}$ & $\mathrm{CH}_{2}$ & $\mathrm{CH}_{3}$ \\
\hline LCP & 1.00 & 1.00 & 1.00 & 1.00 \\
SCP & 0.11 & 0.71 & 0.92 & 0.50 \\
SCPPI & 0 & 0 & -0.25 & 0 \\
LCPD & 0.88 & 0 & 0 & 0.50
\end{tabular}

Figure 7: Matrix of spectral intensities. Ratios of these intensity matrix elements define the coefficients used in the linear combination of spectra to produce the edited subspectra. 
The intensity matrix values define subtraction coefficients to be used in generating the subspectra. Application to the calibration standard, sodium hydroxybutyrate, is shown in Figure 8. Co-addition of the subspectra results in the synthetic CPMAS spectrum, which is essentially identical to an independently acquired CPMAS spectrum as demonstrated by the clean nulling of all resonances in the difference spectrum.

Figure 8: Edited subspectra of Sodium Hydroxybutyrate.

difference
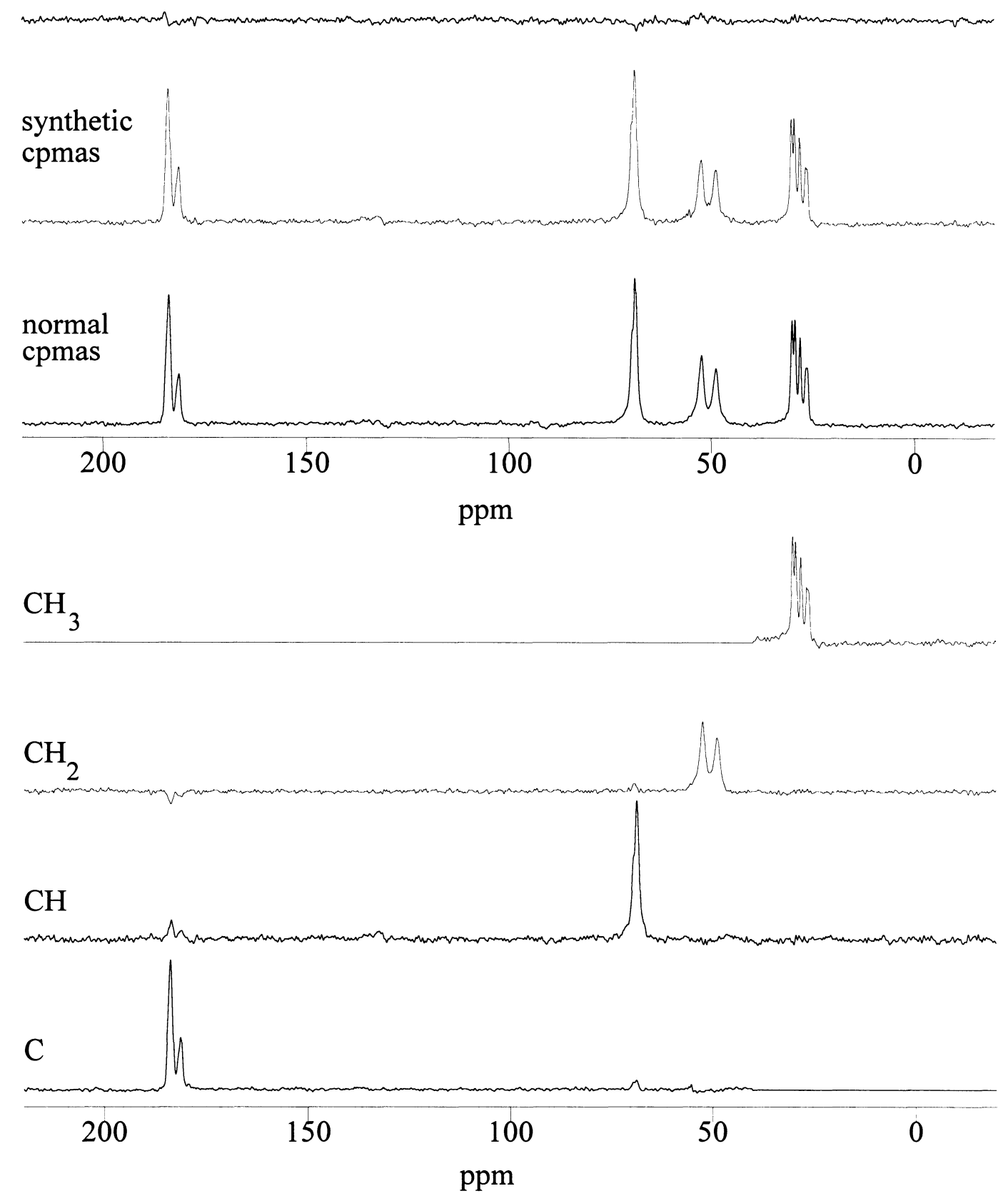
The generality of the intensity matrix obtained for rigid organic solids is demonstrated in application to the editing of the CPMAS spectrum of the enol form of methyl cyclopentanedione. The input data are depicted in Figure 9, while Figure 10 shows the subspectra and a comparison of the synthetic and normal CPMAS spectra.

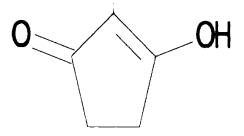

\section{SCPPI}
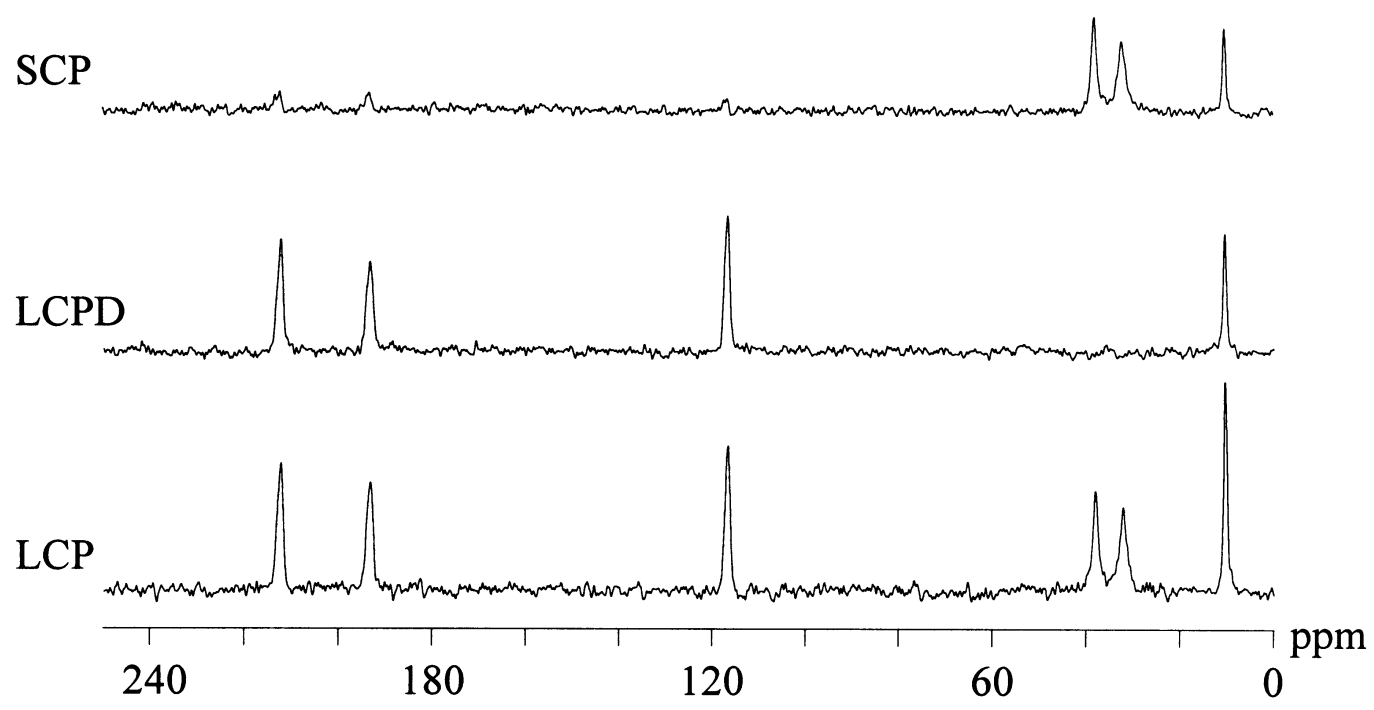

Figure 9: Methyl cyclopentanedione. 


\section{Figure 10: Subspectra of Methyl cyclopentanedione.}

\section{difference}

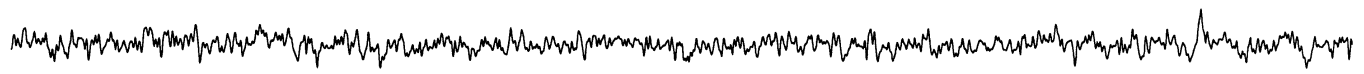
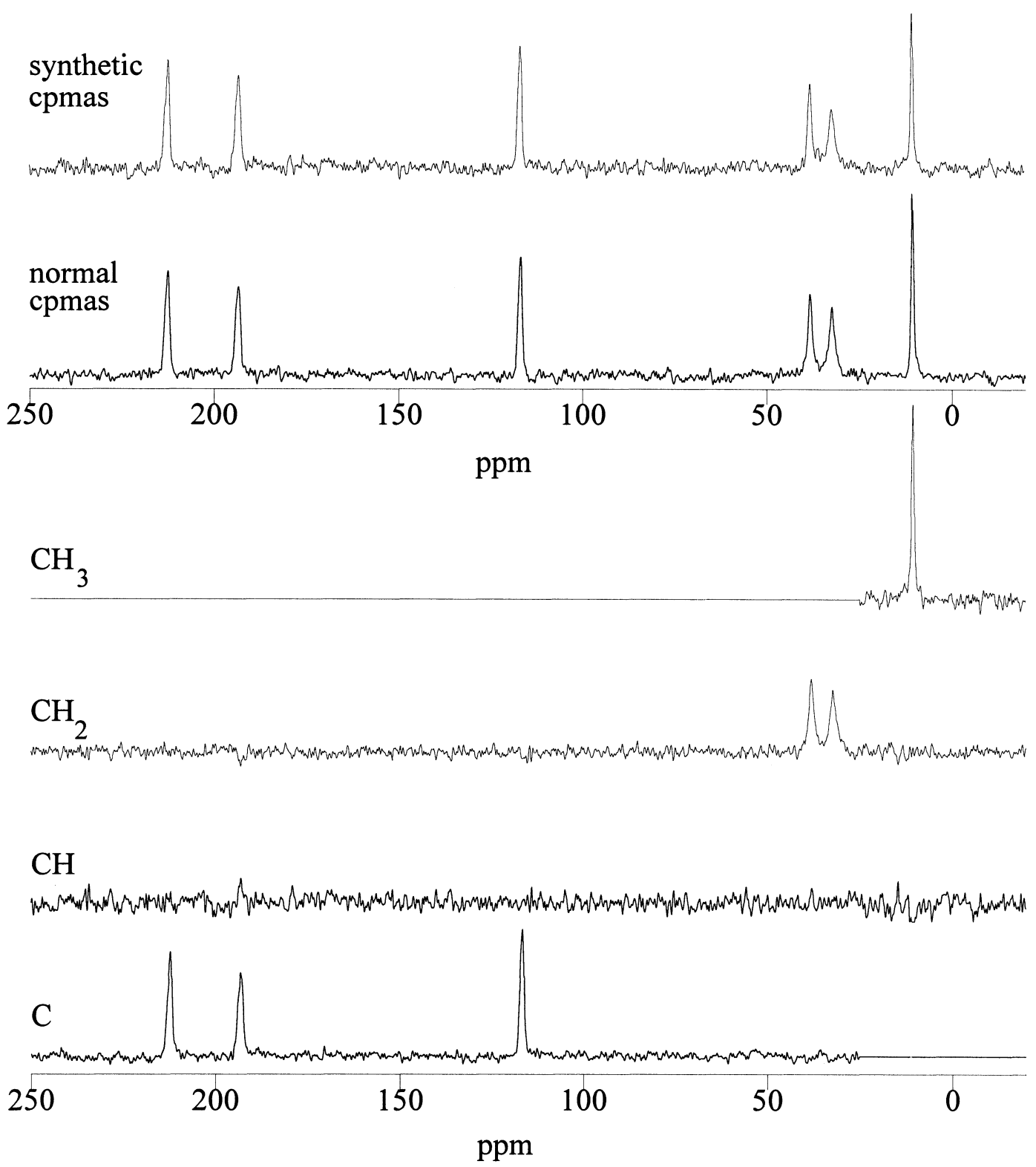

Summary

Although high speed MAS greatly complicates cross polarization dynamics, the differential behavior of carbon centers with different multiplicities is still quite apparent. These differences lend themselves to development of spectral editing protocols for solids NMR which work quite well at MAS rates in excess of $10 \mathrm{kHz}$. While suppression of spin diffusion by magic angle spinlocking can lengthen the time over which the $\mathrm{CP}$ transfer is restricted to tightly coupled ${ }^{13} \mathrm{C}^{1} \mathrm{H}_{\mathrm{n}}$ groups, the oscillatory behavior observed thwarts any potential advantages that might be provided. Work is now in progress to test this new protocol on the Argonne coals. 